\title{
Multidisciplinary and Multisociety Practice Guideline on Reprocessing Flexible Gastrointestinal Endoscopes and Endoscopic Accessories:
}

Korean Association for the Study of the Liver, the Korean Society of Infectious Diseases, Korean College of Helicobacter and Upper Gastrointestinal Research, the Korean Society of Gastroenterology, Korean Society of Gastrointestinal Cancer, Korean Society of Neurogastroenterology and Motility, Korean Association for the Study of Intestinal Diseases, Korean Pancreatobiliary Association, the Korean Society of Gastrointestinal Endoscopy Nurses and Associates and Korean Society of Gastrointestinal Endoscopy

Dae Young Cheung ${ }^{1}$, Byung Ik Jang ${ }^{2}$, Sang Wook Kim³, Jie-Hyun Kim ${ }^{4}$, Hyung Keun Kim', Jeong Eun Shin ${ }^{5}$, Won Jae Yoon ${ }^{6}$, Yong Kang Lee ${ }^{7}$, Kwang Hyun Chung ${ }^{8}$, Soo-Jeong Cho ${ }^{9}$, Hyun Phil Shin ${ }^{10}$, Sun Young Cho ${ }^{11}$, Woon Geon Shin ${ }^{12}$, Kee Don Choi ${ }^{13}$, ByungWook Kim', Joong Goo Kwon ${ }^{14}$, Hee Chan Yang ${ }^{3}$, Tae-Geun Gweon ${ }^{1}$, Hyun Gun Kim ${ }^{15}$, Dong-Won Ahn ${ }^{9}$, Kwang Bum Cho ${ }^{16}$, Sun Hee $\mathrm{Kim}^{17}$, Kyong Hwa Hwang ${ }^{18}$ and Hee Hyuk $\mathrm{Im}^{19}$

${ }^{1}$ Department of Internal Medicine, The Catholic University of Korea College of Medicine, Seoul, ${ }^{2}$ Department of Internal Medicine, Yeungnam University College of Medicine, Daegu, ${ }^{3}$ Department of Internal Medicine, Jeonbuk National University Medical School, Jeonju, ${ }^{4}$ Department of Internal Medicine, Yonsei University College of Medicine, Seoul, ${ }^{5}$ Department of Internal Medicine, Dankook University College of Medicine, Cheonan, ${ }^{6}$ Department of Internal Medicine, Ewha Womans University College of Medicine, Seoul, ${ }^{7}$ Department of Internal Medicine, National Health Insurance Service Ilsan Hospital, Goyang, ${ }^{8}$ Department of Internal Medicine, Eulji University School of Medicine, Daejeon, ${ }^{9}$ Department of Internal Medicine, Seoul National University College of Medicine, Seoul, ${ }^{10}$ Department of Internal Medicine, Kyung Hee University School of Medicine, Seoul, ${ }^{11}$ Department of Internal Medicine, Sungkyunkwan University School of Medicine, Seoul, ${ }^{12}$ Department of Internal Medicine, Hallym University College of Medicine, Seoul, ${ }^{13}$ Department of Internal Medicine, University of Ulsan College of Medicine, Seoul, ${ }^{14}$ Department of Internal Medicine, Daegu Catholic University School of Medicine, Daegu, ${ }^{15}$ Department of Internal Medicine, Soonchunhyang University College of Medicine, Seoul, ${ }^{16}$ Department of Internal Medicine, Keimyung University School of Medicine, Daegu, ${ }^{17} \mathrm{CHA}$ Bundang Medical Center, Sungnam, ${ }^{18} \mathrm{The}$ Catholic University of Korea Seoul St. Mary's Hospital, Seoul, ${ }^{19}$ Soon Chun Hyang University Seoul Hospital, Seoul, Korea

The area of endoscopic application has been continuously expanded since its introduction in the last century and the frequency of its use also increased stiffly in the last decades. Because gastrointestinal endoscopy is naturally exposed to diseased internal organs and contact with pathogenic materials, endoscopy mediated infection or disease transmission becomes a major concern in this field. Gastrointestinal endoscopy is not for single use and the proper reprocessing process is a critical factor for safe and reliable endoscopy procedures. What needed in these circumstances is a practical guideline for reprocessing the endoscope and its accessories which is feasible in the real clinical field to guarantee acceptable prevention of pathogen transmission. This guideline contains principles and instructions of the reprocessing procedure according to the step by step. And it newly includes general information and updated knowledge about endoscopy-mediated infection and disinfection. Multiple societies and working groups participated to revise; Korean Association for the Study of the Liver, the Korean Society of Infectious Diseases, Korean College of Helicobacter and Upper Gastrointestinal Research, the Korean Society of Gastroenterology, Korean Society of Gastrointestinal Cancer, Korean Association for the Study of Intestinal Diseases, Korean Pancreatobiliary Association, the Korean Society of Gastrointestinal Endoscopy Nurses and Associates and Korean Society of Gastrointestinal Endoscopy. Through this cooperation, we enhanced communication and established a better concordance. We still need more researches in this field and fill up the unproven area. And our guidelines will be renewed accordingly. Clin Endosc 2020;53:276-285

Key Words: Endoscope reprocessing; Endoscopy; Guideline

Received: April 21, 2020 Revised: April 27, 2020

Accepted: April 28, 2020

Correspondence: Byung Ik Jang

Department of Internal Medicine, Yeungnam University College of Medicine, 170

Tel: +82-53-620-3831, Fax: +82-53-654-8386, E-mail: jbi@med.yu.ac.kr

Hyeonchung-ro, Nam-gu, Daegu 42415, Korea

ORCID: https://orcid.org/0000-0002-3037-9272

This guideline is being co-published in Clinical Endoscopy (in English) and The Korean Journal of Medicine (in Korean) for the facilitated distribution.

(cc) This is an Open Access article distributed under the terms of the Creative Commons Attribution Non-Commercial License (http://creativecommons.org/licenses/by$\mathrm{nc} / 3.0$ ) which permits unrestricted non-commercial use, distribution, and reproduction in any medium, provided the original work is properly cited. 


\section{INTRODUCTION}

Gastrointestinal (GI) endoscopy is one of the most remarkable innovations in the history of medicine in the 20th century. Since the use of GI endoscopy was started for diagnostic purposes to observe the lumen of the GI tract, this area has expanded to various fields and has advanced such that it is used for the standard treatment of early GI cancer. In addition, the increasing availability and accessibility of the GI endoscopy equipment has played a significant role in public health promotion by enabling the early diagnosis and timely treatment of digestive diseases. In particular, the national cancer screening program in Korea has achieved a dramatic increase in cancer survival rates, ensuring the right to health. Korea's experience in increasing the rates of early diagnosis and reducing mortality rates for gastric and colorectal cancer is the best model, which directly demonstrates the importance of GI endoscopy.

As the distribution and usage of GI endoscopy expands, awareness of the importance of performing and managing GI endoscopy rises. In-hospital infections are a major problem in medical practice, which should be fully considered in the field of GI endoscopy. A GI endoscope is a reusable medical device and is applied to a patient after a reprocessing procedure of cleaning, disinfection, and storage. Activities related to reprocessing such as cleaning and disinfection of GI endoscopes should be recognized as a separate and an essential process and should be allocated a proper budget in each healthcare financial system. There has been a small number of reports on the outbreak or epidemic of GI infections. However, the complicated structure of the duodenoscope and linear-type echoendoscope has the ingrained risk of microbial transmission and was found as the culprit for a fatal antibiotic-resistant bacterial infection in a patient who underwent endoscopic retrograde cholangiography. ${ }^{1,2}$ Therefore, a reliable endoscope reprocessing procedure is the most fundamental condition for safe GI endoscopy. Practical guidelines for the reprocessing of GI endoscopes should be universally applicable in the field and will be effective to prevent the spread of pathogens when adhered to.

The first Korean guideline for endoscope reprocessing was issued by the Korean Society for Gastrointestinal Endoscopy in 1995. It has been revised three times: August 2009, August 2012, and March 2015. The latest revision of 2015 was expanded and improved to include the standards of other countries ${ }^{3-13}$ and had been used as an evaluation criteria for Endoscopy Quality Control by the National Cancer Screening Project and the Certification for Excellence of the Korean Society of Endoscopy. ${ }^{14}$

This 4 th revision was intended to be a comprehensive guideline. It reflects the changes in the medical environment, such as the recent health insurance benefit standards, and incorporates the expertise of the relevant academic societies and working groups, in order to cover not only the cleaning and disinfection aspect but also the knowledge and practice of the management of in-hospital infections and endoscope-transmitted infectious diseases. ${ }^{15}$ To this end, the Korean Association for the Study of the Liver, the Korean Society of Infectious Diseases, Korean College of Helicobacter and Upper Gastrointestinal Research, the Korean Society of Gastroenterology, Korean Society of GI Cancer, Korean Society of Neurogastroenterology and Motility, Korean Association for the Study of Intestinal Diseases, Korean Pancreatobiliary Association, the Korean Society of Gastrointestinal Endoscopy Nurses and Associates and Korean Society of Gastrointestinal Endoscopy participated in this revision. Four workshops were held for all participants. The manuscript was written according to the areas of expertise and the referred literatures were reviewed in common. The details of the revised guidelines were cross-reviewed in a line-by-line manner and was confirmed when all the participants agreed to it. Particularly, the Korean Society of Gastrointestinal Endoscopy Nurses greatly contributed in the aspects of the practical skills and descriptions of the endoscope reprocessing steps. Infection-related issues were nailed to be clear and accurate with the effort of the Korean Society of Infectious Diseases. There are some precedents from other countries, ${ }^{13,16}$ and our revised guideline is the meaningful outcome of strengthened communication and reasonable agreement about practical value from the relevant academic societies and working groups.

This practice guideline for reprocessing the GI endoscope was made based on scientific evidence and expert opinion. This guideline is intended to convey knowledge related to reprocessing GI endoscopes and to provide practical assistance in day-to-day clinical practice. The content of this guideline provides an up-to-date knowledge, but it might not be the absolute standards or principles that should always be applied in every condition. Some of the information contained in this guideline could be an ideal criterion that is still difficult to apply under current conditions, as authors admit that actual implementation will take more effort and time. In the future, this guideline will need to be continuously revised to reflect new knowledge and to respond to the needs of the site and changes in the environment. The guideline consists of healthcare personnel education, knowledge related to reprocessing and infections, and practical skills and instructions in the field.

\section{EDUCATION FOR ENDOSCOPY STAFF}

\section{Endoscopy staff including doctors, nurses, and}


endoscope reprocessing workers should be trained about reprocessing. ${ }^{13,16,17}$

\section{The head of an institution as well as a person in charge of the management of the GI endoscopy department should ensure that endoscope} reprocessing workers receive adequate training. ${ }^{13,16,17}$

Commentary: Because GI endoscopy can mediate the transmission of infections, all endoscopy staff must be educated about the procedure of endoscope reprocessing. The person in charge of the management of the GI endoscopy department should provide continuous education, training, and appropriate management and supervision to endoscope reprocessing practitioners. Endoscope reprocessing practitioners should familiarize themselves with the theory of reprocessing and drill the practice to become proficient in the actual process before working on the site. To this end, relevant organizations or institutions should provide the appropriate training. Education on the theory and practice has the effect of suppressing the occurrence of endoscopy-related infections and preventing endoscopy staff from being exposed to hazards such as infectious agents or harmful substances in the reprocessing procedure. Onetime education and training are not enough and must be continued periodically and regularly.

\section{PROTECTION OF ENDOSCOPY STAFF}

\section{All endoscopy staff including doctors, nurses, and endoscope reprocessing workers should wear appropriate personal protective equipment.}

Commentary: Endoscopy doctors and nurses are required to wear an appropriate personal protective equipment (PPE) because of the high risk of exposure to possibly hazardous materials such as blood, secretions, fecal materials, and bacterial or viral or even fungal microbes during the examination or procedure. ${ }^{7,17}$ Essential PPEs for doctors and nurses are the following: long-sleeved gown with moisture resistant property, protective glasses or visors, face mask, chemical resistant gloves, and clogged and waterproof shoes.

\section{Endoscope reprocessing workers who perform cleaning and disinfection must wear appropriate PPE.}

Commentary: Endoscope reprocessing workers must be protected from various harmful substances from the working environment. In addition to the biological hazardous materials from used endoscopes, the reprocessing environment possibly exposes the worker to chemically noxious materials. Vapor, aerosols, and droplets from disinfectants and various liquids are almost inevitable. Both needles and sharp instruments can also harm the workers. Therefore, reprocessing workers should wear the following PPEs:

- Long-sleeved waterproof gown

- Eye protection (safety glasses or goggles or face protection) and facial mask

- Disposable gloves that do not penetrate or corrode with chemicals

- Clogged waterproof shoes

- Earplugs that can block out noise

- Respiratory protective equipment (when dealing with disinfectants containing components that may irritate the respiratory tract)

\section{GENERAL PRINCIPLES OF THE MANAGEMENT OF INFECTIONS RELATED TO THE ENDOSCOPE REPROCESSING PROCEDURE}

A GI endoscope is a reusable medical device that must be reprocessed every time, including cleaning and disinfection, before being reused in patients. Because the GI endoscope could be exposed to or contaminated with an infectious agent, it is mandatory to prevent the transmission of infection with proper reprocessing. The reprocessing for the GI endoscope requires high-level disinfection, meaning that the disinfectant used in the disinfection step should effectively kill the spores of all microorganisms, including bacteria and viruses, and some bacteria on the surface of the object (Table 1). High-level disinfectant is a type of chemical germicide that serves to inactivate all pathogenic microorganisms on the surface of the endoscope.

\section{Hand hygiene of endoscopy workers}

Hand hygiene must be performed before handling the endoscope, whether or not gloves are used.

Commentary: Endoscopy and surgical procedures present a high risk for exposure to blood or body fluids from patients. Hand hygiene is very vital to reduce the risk of exposure to contaminants and the spread of infection. Handling with a contaminated hand itself contaminates the endoscope. Therefore, hand hygiene must be performed before using the endoscope. $^{18,19}$

\section{Endoscopy-related infection ${ }^{15}$}

\section{Bacterial infection}

When an endoscope-related bacterial infection occurs, the incubation period is relatively short and clinical symptoms 
Table 1. Classification of the Disinfection and Sterilization of Patient-Care Items and Equipment by Spaulding 1,19

\begin{tabular}{lll}
\hline Classification & \multicolumn{1}{c}{ Examples } & Disinfection and sterilization method \\
\hline Critical items & $\begin{array}{l}\text { Objects that enter sterile tissue or the vascular system. This category } \\
\text { includes surgical instruments, cardiac and urinary catheters, implants, } \\
\text { and ultrasound probes used in sterile body cavities, arthroscopes, lapa- } \\
\text { roscopes, electrocautery tips, endo-cervical curettes, transfer forceps and } \\
\text { dental equipment including high speed dental handpieces }\end{array}$ & $\begin{array}{l}\text { Sterilization with steam } \\
\text { Chemical sterilization } \\
\text { Sterilization with hydrogen peroxide } \\
\text { gas plasma }\end{array}$ \\
& $\begin{array}{l}\text { Objects that contact mucous membranes or non-intact skin. This category } \\
\text { includes endoscopes such as gastrointestinal endoscopes, bronchoscopes, } \\
\text { and colonoscopes, respiratory therapy and anesthesia equipment, laryn- } \\
\text { goscope blades, esophageal manometry probes, cystoscopes, anorectal }\end{array}$ & High level disinfection \\
& $\begin{array}{l}\text { manometry catheters, and diaphragm fitting rings, cryosurgical probes, } \\
\text { nasal/anal/vaginal specula, CPR face masks }\end{array}$ & \\
\hline Non-critical items & $\begin{array}{l}\text { Objects that come in contact with intact skin but not mucous membranes. } \\
\text { This category includes bedpans, blood pressure cuffs, crutches and com- }\end{array}$ & Low-level disinfection \\
& puters & \\
\hline
\end{tabular}

$\mathrm{CPR}$, cardiopulmonary resuscitation.

occur early in the infected patient, which can be easily recognized. However, if the symptoms are mild or are related to other factors such as other procedures or the underlying disease of the patient, the transmission through the endoscope may not be recognized.

Examples of bacterial infections transmitted through the endoscope include Salmonella, Pseudomonas, Helicobacter pylori, Klebsiella, Enterobacter, Serratia, and Staphylococcus, and the majority occurs in connection with improper endoscope reprocessing. Recently, cases of carbapenem-resistant Enterobacteriaceae transmission through the duodenoscope have been reported worldwide, including in the United States. The complicated structures (elevator, elevator wire channel, etc.) of the duodenoscope possibly provide a niche for microbial contamination, which cannot be completely reached and controlled with manipulations recommended by the existing endoscope reprocessing guidelines. The US Federal Drug Administration (FDA) has proposed four additional methods in addition to the existing endoscopic reprocessing procedure: monitoring of microbial cultures periodically or for each reprocessing procedure, repeated implementation of standard high-level disinfection, sterilization with ethylene oxide gas, and use of a liquid chemical sterilant reprocessing system. It recommended that one of the four methods should be employed for duodenoscopes according to each institutional situation. Endoscopy manufacturers and related societies have also added separate instructions for cleaning and disinfecting duodenoscopes. Regarding the duodenoscope, we need to keep doing more studies and validations on the reprocessing procedure in order to arrive at a solid resolution. Before then, we should carefully choose the best strategy that fits to the characteristics and reality of each country and institution.

Regarding Mycobacterium infection, we have no evidence of endoscopy-mediated transmission and no reported case. It is well proven that the high-level disinfectant, which is used in endoscope reprocessing, destroys and removes the spores of Mycobacterium tuberculosis and some bacteria, such as Clostridioides difficile.

\section{Viral infection}

Viral infections have a longer incubation period than bacterial infections. The symptoms are often absent or mild, even if present. It could be difficult to confirm the relationship between GI endoscopy and viral infections, but looking at existing data, the possibility of virus transmission through an endoscope is extremely rare.

(1) Hepatitis $\mathrm{B}$ virus (HBV)/hepatitis $\mathrm{C}$ virus (HCV)

A few cases of endoscopy-mediated transmission of HBV and HCV have been reported. However, if proper disinfection guidelines are followed, the possibility of spreading these viruses by endoscopy is extremely low.

(2) Human immunodeficiency virus (HIV)

To date, there has been no report about HIV transmission attributable to GI endoscopy. Manual cleaning with detergents can remove more than $99 \%$ of HIV. High-level disinfection completely removes the virus.

\section{Transmission of other microorganisms}

(1) Parasites

Only Strongyloides infestation has been reported in associa- 
tion with a contaminated GI endoscopy procedure. The transmission of other parasites has not been reported.

\section{(2) Fungi}

The transmission of fungal infections has not been reported in connection to GI endoscopy.

\section{(3) Prion}

The transmission of prion has not been reported in connection to GI endoscopy. Prion is not destroyed by conventional disinfection and sterilization methods. Endoscopic procedures should be avoided for patients with prion infections such as variant Creutzfeldt Jacob disease as much as possible. To date, because single-use endoscopy is not available, it is recommended to use the designated endoscope for the prion-infected patients or to use and discard the endoscope that has reached the end of its life.

\section{Management of endoscopy-related infection outbreak}

Each endoscopy unit should have specific protocols to manage patients and endoscopy staff in case of an endoscopy-related infection outbreak. Suspected devices or suspected problems in the reprocessing procedure should be evaluated according to the established protocol. If any contamination is identified in the endoscope reprocessing procedure, the device should not be used on the patient until appropriate measures have been taken. In the case of an endoscopy-related infection outbreak, a multidisciplinary approach including an endoscopy unit, hospital hygiene-infection expert's board, microbiologists, endoscope manufacturers, and the regulatory board should take part in. ${ }^{17}$

\section{DOCUMENTATION FOR THE ENDOSCOPE REPROCESSING PROCEDURE}

\section{The endoscope reprocessing procedure must} be documented. The documentation should be sufficient to allow the complete recall of the reprocessing procedure carried out for every single endoscopy.

Commentary: Each endoscopy unit has to establish a traceability system regarding the whole sequence of the endoscope reprocessing procedure. The data about the history of endoscopy and the following reprocessing procedure must be stored as a medical record. A traceability system is crucial in the case of an endoscopy-related infection outbreak in terms of epidemiological investigation and patient protection. The following information should be included in the recording section:

- Date of endoscopy
- Information about the examinee (name, registration number, and other identifiers if necessary)

- The type of endoscopic procedure

- The worker who performed the reprocessing procedure

- Reprocessing time

- The serial number of the reprocessed endoscope

- The serial number of the automatic washing sterilizer

The document can be a handwritten book or an electronic medical record. If the information can be identified by electronic records, there is no need to prepare a separate handwritten book.

\section{REGULATIONS OF THE MANAGEMENT OF INFECTION AND STERILIZATION RELATED TO ENDOSCOPE REPROCESSING}

\section{The endoscopy unit should have specific protocols for endoscopy-related infection control and endoscope reprocessing procedures.}

Commentary: To reduce the frequency of infection transmitted through the endoscope and to ensure the safety of the endoscopy staff and reprocessing workers, department-specific protocols for infection control and reprocessing procedure must be established according to the general principles and institutional situation. All endoscope reprocessing procedures and practices for preventing endoscopy-related infections should be conducted, adhering to the detailed instruction and protocols. The Korean Society of Gastrointestinal Endoscopy had published and distributed guidelines for endoscopic reprocessing. Each institution can use or adopt these guidelines.

The department responsible for endoscopy reprocessing needs to monitor whether the reprocessing procedure is being performed according to the protocol. This means that the reprocessing procedure should be carried out consistently, and whether high-level disinfection is maintained must be regularly evaluated according to the recommendations of the guidelines. Besides, it is recommended that the management of automatic endoscope reprocessor (AER): a high-level disinfectant solution, sterilization and disinfection of accessories, and endoscope storage after sterilization ought to be based on the guidelines. Creating and using checklists to ensure that these procedures are done according to the guidelines can help improve the quality control of disinfection.

\section{Intrinsic education to maintain the quality of endoscope disinfection and other reprocessing procedures should be conducted.}


Commentary: Endoscope reprocessing workers need to be familiar with the theory of the reprocessing work and trained to become proficient in the actual process. The institution should provide appropriate training opportunities from either extrinsic or intrinsic sources. If a short-term worker or a new employee cannot complete the endoscopy cleaning/disinfection training conducted by an accredited education provider, an equivalent valued course must be conducted following the training guidelines of the endoscope reprocessing procedure under the supervision of the person in charge of the endoscopy unit management.

\section{Microbial culture examination of the endoscopes and its accessories are recommended to be performed periodically to control the quality of the} reprocessing procedure. In the events of suspected endoscope-mediated infection, a microbial culture study is mandatory. ${ }^{20,21}$

Commentary: Guidelines for endoscope reprocessing recommend the microbial culture test of the endoscope and its accessories for quality control purposes in principle. . $3,16,22^{-2}$ However, specific conditions and principles about the examination interval and culture methods are not yet established. To date, cost-effectiveness analysis of microbial culture is limited. Questions about the interpretation of microbial culture results and the approach to take for culture-positive devices are still not answered. The fundamentals for the microbial culture examination of an endoscope is not strong enough and the recommendation for periodic culture is yet not mandatory. However, for a duodenoscope, which has a complicated structure and possible niches for microbial contamination, periodic microbial culture tests could be helpful to evaluate the appropriateness of the reprocessing procedure. In situations in which an endoscope-mediated infection is suspected, a microbial culture test should be performed for epidemiological investigation. We recommend regular and periodic microbial culture tests according to the infection control regulations of each institution. In particular, duodenoscopes should be monitored with a microbial culture test at least twice a year. Records should be made and stored.

\section{DESIGN FOR SEPARATION AND WORKFLOW OF THE ENDOSCOPE REPROCESSING AREA}

\author{
The room for reprocessing should be separated \\ from the area where endoscopic procedures are \\ performed.
}

Commentary: The endoscopy unit should be designed for proper separation and workflow to minimize the cross- or re-contamination. Particularly, the endoscopy area should be separated from the reprocessing area. In addition, because the hazardous materials including disinfectants, contaminated water, contaminated endoscopes, and accessories are handled in the reprocessing area, improper contact or communication between the reprocessing area and endoscopy area may predispose the exposed patients and endoscopy staffs to risks of biological or chemical injury.

\section{It is recommended to separate the reprocessing area into dirty and clean areas.}

Commentary: The recontamination is an important issue in the reprocessing procedure. Spatial or at least functional flow separation of dirty and clean areas in the reprocessing area has importance in this aspect. ${ }^{1,3}$ One-way workflow can minimize the risk of recontamination of endoscopes and other devices. This also minimizes the risk of patients and endoscopy personnel being exposed to infectious agents and harmful substances.

\section{Adequate ventilation is mandatory in the endoscopy cleaning-disinfection space.}

Commentary: High-level disinfectant solutions are handled in the area where the endoscope is cleaned and disinfected, so adequate ventilation and fume extraction are necessary to ensure that reprocessing workers are not exposed to chemical toxicity. In general, either ventilation systems or fume extraction hoods can be used. A negative pressure ventilation system can further minimize the risk of toxic substances as well as the possibility of infection from contaminated air or particles. $^{4,5}$

\section{It is recommended that the workflow for pre- and post-reprocessing endoscopes should be arranged to be separated.}

Commentary: It is desirable to arrange spaces and transport paths to prevent the risk of re-contamination of the reprocessed endoscope from the contaminated endoscope. It is preferable that the contaminated endoscope is moved to the cleaning-disinfection space through a dedicated path and that the reprocessed endoscope is transferred to the endoscopy room via a separate path. This allows the endoscope to be moved in a one-way workflow. Besides, to prevent the reprocessed endoscope from being contaminated or damaged again during the transportation process, it must be transported in a sterilized sealed container marked "Completed disinfection". The same applies to endoscopic accessories. 
It is recommended that the workers wash their hands in a sink that is different from the sink for cleaning procedures. It is also desirable to have facilities for washing the eyes in case of eye contamination.

\section{STEPS OF THE REPROCESSING PROCEDURE}

The reprocessing procedure consists of six stages: pre-cleaning, cleaning, disinfection, rinsing, drying, and storage. The principles and practices of each stage are presented below.

\section{Pre-cleaning (bedside cleaning)}

After the endoscope has been withdrawn from the patient, the surface of the endoscope should be cleaned with gauze soaked with detergent or sterile distilled water. Pre-cleaning should be done immediately next to the bedside.

Commentary: The detergent contains slightly different ingredients depending on the manufacturer. It functions to effectively break down contaminants from the human body, such as blood, secretions, polysaccharides, lipids, and biofilms. At the same time, detergents should be compatible with the endoscopes and their accessories. Enzyme-containing detergents are more effective in the removal of proteins.

The tip of the endoscope should be put in the cleaning solution and the cleaning solution aspirated into the working channel to remove contaminants inside.

Commentary: Aspirate the cleaning solution and air in an alternating pattern. This maneuver can remove contaminants in the working channel more effectively than aspiration of cleaning solution alone. If this process is not performed immediately after the end of the endoscopy procedure, contaminants such as body fluids and blood in the narrow channel will solidify, and the subsequent disinfection process will not completely remove the contaminants. Finally, after aspirating air into the channel and making it empty, the endoscope can be separated from the endoscope system.

After separating the endoscope from the system, a precleaned endoscope should be transported in a dedicated container to the reprocessing area. During transportation, it is better to cover the lid of the container and carry it.

\section{Cleaning}

Remove all removable/detachable parts from the endoscope.
Commentary: Cleaning is a manual process. On the handle of the endoscope, there are removable/detachable parts including a suction valve, air valve, biopsy channel valve. On the tip of the endoscope, there could be a distally attached transparent cap or balloon in case of echoendoscopes. All parts should be removed and then cleaned separately and thoroughly.

\section{Leak test}

Commentary: Leak test is to check for damage inside and outside the endoscope and is performed according to the manufacturer's instructions. If moisture is filled inside the endoscope, it may cause a breakdown and corrosion. To check for leakage, connect the endoscope to the washer body or use a leak test equipment. The entire endoscope is placed in a container filled with water and observed for 30 seconds. During this period, watch carefully whether air bubbles continuously come out from the tip, suction valve, or biopsy channel. In case of any detectable leakage, the reprocessing procedure must be interrupted. The damaged endoscope must be handed to the repair professional or repair center immediately. Leak inspection should always be performed at the beginning of the reprocessing procedure. Cleaning with water or detergent possibly increases the damage of the device when it leaks, so it is necessary to check for any abnormality before proceeding to the cleaning step.

Wash the endoscope with a cleaning solution, and wash and brush all parts separated from the biopsy channel and the endoscope handle.

Commentary: The cleaning solution effectively penetrates contaminants composed of proteins, fats, carbohydrates, and various chemical bases so that the contaminants can easily fall inside the channel. At the same time, it should be compatible with the endoscope and its accessories. It is recommended to use an enzyme cleaner as a cleaning solution. Given that the endoscope has no damage and no leak, it can be immersed in an adequately sized sink with the cleaning solution and then the surface is wiped with a soft cloth and sponge. Contaminants inside the channel and forceps are completely removed using a brush that fits the endoscope channel. A disposable brush, cloth, or sponge is recommended as much as possible, but if not possible, a reusable one can be used after sterilization. All the parts separated from the biopsy channel and the endoscope should be cleaned using a brush.

(1) In the case of an endoscope with an elevator, such as a duodenoscope and a linear-type echoendoscope, the parts of the elevator and brush should be thoroughly cleaned on every side with up and down manipulation of the elevator so that no foreign materials remain. ${ }^{23}$

Commentary: The duodenoscope and the linear-type echo- 
endoscope have an elevator for directional manipulation of the forceps or needles at the tip of the endoscope. The elevator makes the exposed end of the endoscope complicated. Also, because of the clearance for the operation of the device, there is a small space and a gap at the tip, such that organic matter or contaminants are likely to remain in this space. Because the elevator device and the wire channel are difficult to be cleaned with an AER, hand washing must be thoroughly performed by injecting cleaning solution, water, and air several times according to the manufacturer's instructions for each endoscope.

The ultrasonic cleaner is effective to clean some sturdy accessories that are not suitable for hand brushing and AER.

Wash the remaining cleaning solution thoroughly with clean water (even when using an AER, the cleaning step should be performed manually).

\section{Disinfection}

High-level disinfectants are used to disinfect the endoscope during the reprocessing procedure. The high-level disinfectant should be used following the specified conditions such as the effective concentration, application time, and expiration date (and operating temperature) suggested by the manufacturer.

Commentary: The high-level disinfectant solution must be approved and certified for endoscope disinfection by the FDA and must comply with the standards of the Ministry of Health and Welfare. It is crucially important to adhere to the conditions and application time of the disinfectant provided by the manufacturer. The concentration of disinfectant should be checked to ensure that the effective concentration is maintained properly. Currently, $2 \%$ glutaraldehyde, $0.55 \%$ ortho-phthalaldehyde, $7.5 \%$ or more hydrogen peroxide, $0.2 \%$ or more peracetic acid (or a mixture of hydrogen peroxide and peracetic acid), and electrolytic acid water are the generally approved disinfectants for the GI endoscope reprocessing. ${ }^{15,17,24-26}$

When disinfection is carried out manually instead of using an AER, the use of an appropriately sized container is important not to corrode the endoscope in the disinfecting solution. Immerse the endoscope and appendages completely in the high-level disinfectant solution and fill disinfectants repeatedly in each channel so that there is no empty space.

Commentary: Manual disinfection requires a sufficiently big container to avoid twisting the endoscope. Also, the endoscope should not be carried with sharp tools that can damage it. The endoscope should be completely immersed in the disinfectant solution and the biopsy channel should be instilled with the disinfectant solution using a syringe or a similar instrument. If there is a space where the disinfectant solution does not penetrate, microbes can multiply and grow at the part of endoscope, thus disinfection will not be completely achieved. Therefore, it must be instilled slowly and filled up to prevent air bubbles from the brisk injection. Most high-level disinfectants are prepared for multiple usages, but the concentration decreases with usage and the effect of disinfection decreases with time after opening. It is important to check the minimum effective concentration that can maintain the effect of disinfection. Before the first disinfection process of each day, the concentration of the disinfectant is checked. If the concentration of disinfectant measured is under the minimum effective concentration, it must be discarded. Further, the disinfectant beyond the expiration date must also be discarded, regardless of whether the minimal effective concentration is maintained or not. It is desirable to use a timer to comply with the immersion time.

In case of using an AER, the disinfection should be performed according to the manufacturer's manual.

Commentary: AERs are recommended because they have the advantage of being able to undergo an automated and a consistent disinfection process and having a low risk of exposure of the disinfectant to endoscopy reprocessing practitioners.

\section{Rinsing}

Using "sufficiently clean water", thoroughly rinse the endoscope and various valves (biopsy channel valve, suction valve, air valve).

Commentary: Rinsing is a process of removing any disinfectant that may remain in the endoscope. Water quality is very important. "Sufficiently clean water" means sterilized or filtered water through the purification process. It must be above the edible level and be free from any microbes. Because the residual disinfectant can injure the skin and mucous membranes, thoroughly rinse the endoscope and the interior parts of each channel with a "sufficient" amount of clean water. For example, at least $150 \mathrm{~mL}$ of water is needed to rinse one channel inside the endoscope.

\section{Drying}

Wipe the endoscope surface with a clean cloth and remove the remaining water inside the channel by blowing compressed air and $70 \%-90 \%$ ethyl alcohol or isopropyl alcohol through the channel.

Commentary: Bacteria such as Pseudomonas aeruginosa can multiply in moist areas, so drying after disinfection is an 
important process to prevent contamination. The endoscope surface should be wiped off with a clean cloth to leave no moisture and then the alcohol flow through the channel to dry up. Compressed air is useful, but care must be taken because excessively high pressure may damage the inner channel of the flexible endoscope.

\section{Storage of endoscopes}

Store the endoscopes in a specialized storage cabinet with a controlled environment system. Hang endoscopes vertically in the storage cabinet high enough not to make the tip touch the floor. If horizontal storage is chosen, the storage cabinet should be equipped with a system of continuous air supply to the endoscope channels to ensure its dryness.

Commentary: To prevent the recontamination of the reprocessed endoscope, the storage cabinet should be kept clean and dust-free and better separated from the cleaning and disinfecting area. All detachable parts of the endoscope should be removed and stored without connection. The channels must be uncapped and stored without the biopsy forceps valve, suction valve, air valve, and the waterproof cap. The storage cabinet should be cleaned by wiping with environmental disinfectant daily.

\section{Endoscopic accessories}

The accessories used in the endoscope can be divided into attachable devices and insertion devices into the biopsy channel.

Reprocessing must be applied only to reusable accessories.

Remove it from the endoscope, soak it in the cleaning solution, and wipe it well with a brush and sponge to clean the inside of all the accessories.

The ultrasonic cleaner should be used to remove tiny contaminants adhered to or stuck in a slit of the accessories.

Rinse with clean water and dry with a clean cloth and compressed air.

Depending on the type of accessories, sterilize or disinfect based on the method recommended by each manufacturer before storing it. Suction valves and air valves are reusable accessories and can be used after high-level disinfection. The " $O$ " ring inside the suction valve and the air valve can be worn out after usage or reprocessing procedure, it should be checked up and replaced if needed. Biopsy channel rubber valves with reusable properties can also be used after proper disinfection. It can be worn out after multiple usages and should be replaced. Recently, single-use biopsy channel valves are available.

Commentary: The representative treatment accessory devices include biopsy forceps, loop snare, guidewire, and balloon expander. Invasive devices that penetrate the epithelial barrier of the GI tract should be sterilized. If available, single-use devices are recommended for safety.

Single-use devices must be disposed of after usage and must not be reprocessed for reuse.

Commentary: Consideration should be given to the loss of function of devices after the reprocessing procedure. Apart from the safety issue of disinfection and sterilization, durability and functional adequacy of accessory devices are very important. Devices can be worn out with multiple usages as well as with the reprocessing procedure, even with reusables. ${ }^{27}$ The cost of reprocessing is another aspect to be considered. Whether to choose single-use or reusable devices, we need more consideration on the costs, safety, functional consistency, and convenience in facility management. In recent studies about the functional efficacy of biopsy forceps, researchers advocated for the disposable biopsy forceps in terms of tissue acquisition over the reusables. The single-use biopsy forceps have come to be preferred in most institutions nowadays.

\section{Water bottle and connecting devices}

The water bottle and connecting device should be sterilized or disinfected at a high level once a day. Water in the bottle should be the sterilized once.

Commentary: There are no data on the optimal exchange cycle, stability and potential risks of water bottles, lens cleansing water, connecting and suction tubes, and vacuum containers for waste storage. However, for safety, we recommend high-level disinfection or sterilization of these devices mentioned above at least once a day.

\section{CONCLUSIONS}

GI endoscopy plays a key role in the diagnosis and treatment of diseases of the GI tract, pancreas, and biliary tract. The range and frequency of its use are constantly expanding. Because the GI endoscope is directly exposed to and in contact with the GI epithelial barrier and possible pathogenic substances within the human body, there is a risk of mediating harmful substances or infectious agents. In this reprocessing guideline, general considerations of the cleaning and disinfection process were included. The principles of practice and 
the step-by-step technical skills were described to meet the need of the real site. This reprocessing guideline is the fourth revision since the first one in 1995 by the Korean Society of Gastrointestinal Endoscopy. This guideline is distinguished not only in its content but also in its writing process involving a multidisciplinary and multi-society collaboration. Through this multidisciplinary and multi-society work, it was possible to strengthen communication between experts in each field and to reach agreements and to complement each other on different areas of understanding. Guidelines are not an eternal truth, and thus, outcomes and evidence from future studies and practices will continue to revise the existing guidelines and manuals.

\section{Conflicts of Interest}

The authors have no financial conflicts of interest.

\section{ORCID}

Dae Young Cheung: https://orcid.org/0000-0003-4150-3555

Sang Wook Kim: https://orcid.org/0000-0001-8209-540X Jie-Hyun Kim: https://orcid.org/0000-0002-9198-3326 Hyung Keun Kim: https://orcid.org/0000-0003-3138-0141 Jeong Eun Shin: https://orcid.org/0000-0001-5706-3967 Won Jae Yoon: https://orcid.org/0000-0003-2292-1348 Yong Kang Lee: https://orcid.org/0000-0003-2929-4447 Kwang Hyun Chung: https://orcid.org/0000-0002-8376-3921

Soo-Jeong Cho: https://orcid.org/0000-0001-7144-0589 Hyun Phil Shin: https://orcid.org/0000-0002-9350-1079 Sun Young Cho: https://orcid.org/0000-0001-9307-2369 Woon Geon Shin: https://orcid.org/0000-0002-9851-5576 Kee Don Choi: https://orcid.org/0000-0002-2517-4109 Byung-Wook Kim: https://orcid.org/0000-0002-2290-4954 Joong Goo Kwon: https://orcid.org/0000-0003-3392-785X Hee Chan Yang: https://orcid.org/0000-0001-9847-5343 Tae-Geun Gweon: https://orcid.org/0000-0002-0884-7228 Hyun Gun Kim: https://orcid.org/0000-0001-7545-4638 Dong-Won Ahn: https://orcid.org/0000-0002-6641-2177 Kwang Bum Cho: https://orcid.org/0000-0003-2203-102X Sun Hee Kim: https://orcid.org/0000-0002-2978-561X Kyong Hwa Hwang: https://orcid.org/0000-0003-4168-8414 Hee Hyuk Im: https://orcid.org/0000-0003-4429-5069

\section{REFERENCES}

1. Rutala WA, Weber DJ. Outbreaks of carbapenem-resistant Enterobacteriaceae infections associated with duodenoscopes: what can we do to prevent infections? Am J Infect Control 2016;44(5 Suppl):e47-e51.

2. Oh IH, Son BK. [Duodenoscope-associated infections: a literature review and update]. Korean J Pancreas Biliary Tract 2018;23:145-149.

3. Korea Centers for Disease Control and Prevention. Sterilization and disinfection guidelines in the medical institution. Cheongju: KCDC; 2014.

4. Ministry of Health and Welfare. Guidelines for medical instruments and supplies. Sejong: MOHW; 2010.

5. Cho YS. Importance of endoscope reprocessing and new guideline for reprocessing. In: The 52th Seminar of Korean Society of Gastrointestinal Endoscopy; 2015 Mar 29; Goyang, Korea. Seoul: Korean Society of Gastrointestinal Endoscopy; 2015. p. 338-342.

6. The Disinfection Management Committee of Korean Society of Gas- trointestinal Endoscopy. Guidelines for disinfection of gastrointestinal endoscopes. Seoul: Medbook; 2012.

7. Hookey L, Armstrong D, Enns R, Matlow A, Singh H, Love J. Summary of guidelines for infection prevention and control for flexible gastrointestinal endoscopy. Can J Gastroenterol 2013;27:347-350.

8. Lim CH, Choi MG, Kim WC, et al. Performance and cost of disposable biopsy forceps in upper gastrointestinal endoscopy: comparison with reusable biopsy forceps. Clin Endosc 2012;45:62-66.

9. Oh HJ, Kim JS. Clinical practice guidelines for endoscope reprocessing. Clin Endosc 2015;48:364-368.

10. Oh KJ. Reprocessing and maintaining of endoscope accessories. In: The 50th Seminar of Korean Society of Gastrointestinal Endoscopy; 2014 Mar 30; Goyang, Korea. Seoul: Korean Society of Gastrointestinal Endoscopy; 2014. p. 330-334.

11. Son BK. Disinfectants for endoscope reprocessing. In: The 50th Seminar of Korean Society of Gastrointestinal Endoscopy; 2014 Mar 30; Goyang, Korea. Seoul: Korean Society of Gastrointestinal Endoscopy; 2014. p. 327-329.

12. Yoon IJ. Tips for safe endoscopic reprocessing. In: The 49th Seminar of Korean Society of Gastrointestinal Endoscopy; 2013 Aug 25; Goyang, Korea. Seoul: Korean Society of Gastrointestinal Endoscopy; 2013. p. 324-327.

13. Reprocessing Guideline Task Force, Petersen BT, Cohen J, et al. Multisociety guideline on reprocessing flexible GI endoscopes: 2016 update. Gastrointest Endosc 2017;85:282-294.e1.

14. Kim BW, Son BK, Kim WH, et al. [Korean Society of Gastrointestinal Endoscopy (KSGE) guidelines for endoscope reprocessing]. Korean J Med 2017;92:239-244.

15. Korea Centers for Disease Control and Prevention. Standard prevention guideline for healthcare-associated infection. Cheongju: KCDC; 2017.

16. Beilenhoff U, Biering H, Blum R, et al. ESGE-ESGENA technical specification for process validation and routine testing of endoscope reprocessing in washer-disinfectors according to EN ISO 15883, parts 1, 4, and ISO/TS 15883-5. Endoscopy 2017;49:1262-1275.

17. Beilenhoff $\mathrm{U}$, Biering $\mathrm{H}$, Blum R, et al. Reprocessing of flexible endoscopes and endoscopic accessories used in gastrointestinal endoscopy: position statement of the European Society of Gastrointestinal Endoscopy (ESGE) and European Society of Gastroenterology Nurses and Associates (ESGENA) - update 2018. Endoscopy 2018;50:1205-1234.

18. World Health Organization. WHO guidelines on hand hygiene in health care. Geneva: WHO; 2009.

19. Korea Centers for Disease Control and Prevention. Guideline for hand hygiene in healthcare facilities. Cheongju: KCDC; 2014.

20. Higa JT, Choe J, Tombs D, Gluck M, Ross AS. Optimizing duodenoscope reprocessing: rigorous assessment of a culture and quarantine protocol. Gastrointest Endosc 2018;88:223-229.

21. Kovaleva J. Infectious complications in gastrointestinal endoscopy and their prevention. Best Pract Res Clin Gastroenterol 2016;30:689-704.

22. Petersen BT, Chennat J, Cohen J, et al. Multisociety guideline on reprocessing flexible GI endoscopes: 2011. Infect Control Hosp Epidemiol 2011;32:527-537.

23. The Disinfection Management Committee and Endoscopic Sedation Committee of Korean Society of Gastrointestinal Endoscopy. Guideline handbook for endoscope cleaning and disinfection. Seoul: KSGE; 2017.

24. Ministry of Health and Welfare. Guideline for disinfection of devices and items in healthcare facilities. Sejong: MOHW; 2017.

25. Rutala WA, Weber DJ, Healthcare Infection Control Practices Advisory Committee (HICPAC). Guideline for disinfection and sterilization in healthcare facilities, 2008 [Internet]. Reston (VA): Practice Greenhealth; c2008 [updated 2019 May; cited 2020 May 28]. Available from: https:// practicegreenhealth.org/tools-and-resources/cdc-guideline-disinfection-and-sterilization-healthcare-facilities.

26. Korea Centers for Disease Control and Prevention. Guideline for disinfection and sterilization in healthcare facilities. Cheongju: KCDC; 2014

27. Fireman Z. Biopsy forceps: reusable or disposable? J Gastroenterol Hepatol 2006;21:1089-1092. 